\title{
Analytic approach to the motion of cosmological phase transition fronts
}

\author{
Ariel Mégevand* and Alejandro D. Sánchez ${ }^{\dagger}$ \\ IFIMAR (CONICET-UNMdP), \\ Departamento de Física, Facultad de Ciencias Exactas y Naturales, UNMdP, \\ Deán Funes 3350, (7600) Mar del Plata, Argentina
}

\begin{abstract}
We consider the motion of planar phase-transition fronts in first-order phase transitions of the Universe. We find the steady state wall velocity as a function of a friction coefficient and thermodynamical parameters, taking into account the different hydrodynamic modes of propagation. We obtain analytical approximations for the velocity by using the thin wall approximation and the bag equation of state. We compare our results to those of numerical calculations and discuss the range of validity of the approximations. We analyze the structure of the stationary solutions. Multiple solutions may exist for a given set of parameters, even after discarding nonphysical ones. We discuss which of these will be realized in the phase transition as the stationary wall velocity. Finally, we discuss on the saturation of the friction at ultra-relativistic velocities and the existence of runaway solutions.
\end{abstract}

\section{Introduction}

In a first-order cosmological phase transition, bubbles nucleate and expand, converting the high-temperature phase into the low-temperature one (see, e.g., [1, 2, 3]). As bubbles expand, latent heat is released at their boundaries. This energy raises the temperature and causes bulk motions of the plasma. The perturbations caused in the cosmic fluid by the nucleation and expansion of bubbles generate a departure from thermal equilibrium. This may give rise to a number of cosmic relics, such as a baryon number asymmetry [4], baryon inhomogeneities [5], magnetic fields [6], topological defects [7], or gravitational waves [8, 9].

In general, the system can be described by a relativistic fluid and a scalar field $\phi$ at finite temperature $T$ [10, 11, 12]. The latter may be a Higgs field and acts as an order

\footnotetext{
*Member of CONICET, Argentina. E-mail address: megevand@mdp.edu.ar

${ }^{\dagger}$ Member of CONICET, Argentina. E-mail address: sanchez@mdp.edu.ar
} 
parameter. At high temperatures the free energy $\mathcal{F}(\phi, T)$ has a minimum $\phi_{+}(T)$ (in general, $\left.\phi_{+} \equiv 0\right)$ and at low temperatures a different minimum $\phi_{-}(T)$. In a first-order phase transition, the two minima coexist in certain range of temperatures, separated by a barrier. In the high- $T$ phase, the free energy density is given by $\mathcal{F}_{+}(T)=\mathcal{F}\left(\phi_{+}(T), T\right)$, whereas in the low- $T$ phase, it is given by $\mathcal{F}_{-}(T)=\mathcal{F}\left(\phi_{-}(T), T\right)$. The critical temperature $T_{c}$ is that for which $\mathcal{F}_{+}\left(T_{c}\right)=\mathcal{F}_{-}\left(T_{c}\right)$. The phase transition occurs when the temperature of the Universe reaches $T_{c}$. At $T=T_{c}$, though, the nucleation rate vanishes, and bubbles effectively begin to nucleate at some temperature $T_{n}$ below $T_{c}$ [2, 13].

The nucleated bubbles expand due to the pressure difference between the two phases. In general, the bubble walls reach a terminal velocity $v_{w}$ due to the friction with the surrounding plasma. Recently, the hydrodynamics of the moving walls has received much attention (see, e.g., [14, 15, 16, 17, 18]) due to the interest in performing thorough calculations of the wall velocity and the energy injected into bulk motions of the fluid. These quantities are relevant for the generation of baryons and gravitational waves. In Ref. [15], the ultra-relativistic velocity regime was considered, and it was shown that a state of continuous acceleration of the bubble wall is possible. Such "runaway" solutions may play an important role in the generation of gravitational waves.

A realistic evaluation of the cosmological consequences of a phase transition requires considering the dynamics as completely as possible. Following the development of a phase transition involves the calculation of several temperature-dependent quantities such as, e.g., the pressure of the two phases and the bubble nucleation rate. During the phase transition the temperature varies in time and space due to the adiabatic cooling of the Universe and the release of latent heat. As a consequence, one has to deal with a set of integro-differential equations. Some of the involved variables are very sensitive to approximations (for instance, the nucleation rate). In order to avoid large errors, it is convenient to resort to nontrivial numerical calculations for these quantities. On the other hand, finding analytical approximations for other variables (e.g., the bubble wall velocity) provides a way of reducing the computation time. Widely used simplifications include the thin wall approximation and the bag equation of state [10, 11, 12, 19]. Even with these approximations, it is not always possible to obtain analytical results.

A considerable simplification is achieved by considering planar walls. Analytic results for the planar case were found recently in Ref. [14] for the wall velocity and in Ref. [17] for the energy injected into the fluid. It is important to note that considering spherical bubbles is not necessarily a better approximation than considering planar walls. Although the spherical symmetry is a good approximation for the initial stages of bubble growth, some cosmologically interesting outcomes of the phase transition are produced when bubbles collide and lose the spherical symmetry. Moreover, losing the spherical symmetry is a requirement, e.g., for the generation of turbulence or of gravitational waves. As an explicit example, the "envelope approximation" for the generation of gravitational waves in bubble collisions neglects the overlap regions of colliding bubbles and follows only the evolution of the uncollided bubble walls. For such a calculation, the approximation of treating the walls as planar is, in principle, as good as considering spherical bubbles (but less complicated). In general, one does not expect important differences (see Ref. [17] for 
a comparison of different wall geometries).

In this paper we investigate the propagation of a planar phase transition front in the plasma. We aim at finding analytical approximations for the stationary velocity of the bubble wall as a function of the friction and the thermodynamical parameters. The present work is a continuation of the investigations of Ref. [14], where we considered planar walls propagating as weak deflagrations or weak detonations. Here we include into consideration the case of supersonic Jouguet deflagrations [11] and the possibility that the walls run away (see Ref. [16] for a recent study for spherical-symmetry walls). We also discuss here whether the different solutions are physical or not, and which of them will be realized as final stationary states during the phase transition. We discuss on the validity of the analytical approximations. The approximations are better for weaker solutions than for those close to the Jouguet points. For comparison, we consider some cases previously studied with numerical calculations [10, 11, 12].

The paper is organized as follows. In section 2 we consider the equations for the profiles of the fluid and the bubble wall, including a phenomenological friction term. We study the thin wall limit. In section 3 we use the bag equation of state to obtain a set of analytic equations for the wall velocity. In sections 4 and 5 we present our results for the stationary motion and compare them with those of the numerical works of Refs. [10, 11, 12]. In section 4 we discuss the range of validity of the analytical approximations and in section 5 we analyze the dependence of the wall velocity on the thermodynamic parameters and the friction. In section 6 we consider a different phenomenological friction term, which takes into account the fact that, in some models, the friction force approaches a constant in the ultra-relativistic regime. Finally, we conclude in section 7.

\section{$2 \quad$ Hydrodynamics and microphysics}

We shall consider the motion of bubble walls at a given temperature $T_{n}<T_{c}$, i.e., we shall regard the nucleation temperature $T_{n}$ as a free parameter. Therefore, we shall not be concerned here with the calculation of the amount of supercooling. All the thermodynamic quantities (energy density, pressure, temperature, etc.) are derived from the free energy density. We have two phases, characterized by the minima $\phi_{ \pm}$. Thus, the equation of state (EOS) is different in each phase. For instance, the energy density is given by $\rho_{ \pm}(T)=\mathcal{F}_{ \pm}(T)-T \mathcal{F}_{ \pm}^{\prime}(T)$, where a prime indicates derivative with respect to $T$. The pressure is given by $p_{ \pm}(T)=-\mathcal{F}_{ \pm}(T)$. The enthalpy density is given by $w_{ \pm}=\rho_{ \pm}+p_{ \pm}$, and the entropy density by $s_{ \pm}=w_{ \pm} / T$. The speed of sound is given by $c_{ \pm}^{2}(T)=\partial p_{ \pm} / \partial \rho_{ \pm}=$ $p_{ \pm}^{\prime}(T) / \rho_{ \pm}^{\prime}(T)$. The latent heat is defined as $L \equiv \rho_{+}\left(T_{c}\right)-\rho_{-}\left(T_{c}\right)$.

In the regions separating the two phases the scalar field $\phi$ varies from $\phi_{+}$to $\phi_{-}$. These interfaces are the walls of expanding bubbles, i.e., the phase-transition fronts. In general, temperature gradients arise during the phase transition, and the temperature varies beyond the bubble walls. Thus, the system is characterized by the scalar field $\phi(\mathbf{x}, t)$, the temperature $T(\mathbf{x}, t)$ of the plasma (which we treat as a perfect relativistic 
fluid), and the fluid velocity $v(\mathbf{x}, t)$. These variables are governed by the equations

$$
\begin{aligned}
\partial_{\mu}\left(-T \frac{\partial \mathcal{F}}{\partial T} u^{\mu} u^{\nu}+g^{\mu \nu} \mathcal{F}\right)+\partial_{\mu} \partial^{\mu} \phi \partial^{\nu} \phi & =0 \\
\partial_{\mu} \partial^{\mu} \phi+\frac{\partial \mathcal{F}}{\partial \phi}+\tilde{\eta} T f(\phi / T) u^{\mu} \partial_{\mu} \phi & =0
\end{aligned}
$$

where $u^{\mu}=(\gamma, \gamma \mathbf{v})$ is the four-velocity field of the fluid, with $\gamma=1 / \sqrt{1-v^{2}}$. Equation (1) follows from energy-momentum conservation, $\partial_{\mu} T^{\mu \nu}=0$, whereas Eq. (21) is the equation of motion for $\phi$, where we have introduced a general phenomenological damping term proportional to $\partial_{\mu} \phi$ in order to account for the friction force acting on the scalar field. In section 6 we shall consider a modification of this term which does not grow with $\gamma$ for large $\gamma$. The function $f$ and the dimensionless friction parameter $\tilde{\eta}$ can be derived by considering the microphysics in specific models [14, 20, 21].

We are interested in the steady-state motion of bubble walls. Therefore, we assume stationary profiles moving with the wall at constant velocity. We shall consider planarsymmetry fronts moving in the $x$ direction. In the rest frame of the front, all time derivatives vanish and Eqs. (1+2) become

$$
\begin{gathered}
-T \frac{\partial \mathcal{F}}{\partial T} \gamma^{2} v=\text { constant } \\
-T \frac{\partial \mathcal{F}}{\partial T} \gamma^{2} v^{2}-\mathcal{F}+\frac{1}{2}\left(\frac{d \phi}{d x}\right)^{2}=\text { constant } \\
\frac{d^{2} \phi}{d x^{2}}-\frac{\partial \mathcal{F}}{\partial \phi}-\tilde{\eta} T f(\phi / T) \frac{d \phi}{d x} \gamma v=0 .
\end{gathered}
$$

For a given model the static, 1-dimensional Eqs. (345) or the dynamic, 4-dimensional equations (112), can be integrated numerically, e.g., using a lattice [10, 11, 12]. However, it is very useful to assume that the interface is infinitely thin, thus eliminating the scalar field profile from hydrodynamics considerations. Assuming a thin wall is in general a good approximation, as the wall width is much smaller than the width of the fluid profiles. Indeed, the latter is determined by the dynamics of the phase transition and is roughly given by the time scale $t$. In contrast, the width of the wall, determined by the characteristic length of variation of $\phi$, is roughly given by the scale $T^{-1}$. In general, $t$ is many orders of magnitude larger than $T^{-1}$. As we shall see, taking the thin wall limit in Eqs. (3,4 is trivial, whereas doing so in Eq. (5) requires additional approximations.

\subsection{Hydrodynamics}

Equations (3) and (44) relate the fluid variables on each side of the wall (in the rest frame of the wall). We shall use a + sign for variables just in front of the wall and a - sign for variables just behind the wall. Since $\phi^{\prime}$ vanishes outside the interface, we have

$$
\begin{aligned}
w_{-} v_{-} \gamma_{-}^{2} & =w_{+} v_{+} \gamma_{+}^{2}, \\
w_{-} v_{-}^{2} \gamma_{-}^{2}+p_{-} & =w_{+} v_{+}^{2} \gamma_{+}^{2}+p_{+} .
\end{aligned}
$$


These equations give $v_{+}$as a function of $v_{-}$. The solutions have two branches (see Fig. 1), called detonations and deflagrations. For detonations the incoming flow is faster than the outgoing flow $\left(\left|v_{+}\right|>\left|v_{-}\right|\right)$. The value of $\left|v_{+}\right|$is supersonic in all the range $0<\left|v_{-}\right|<1$, and has a minimum at the Jouguet point $\left|v_{-}\right|=c_{-}$. The minimum value of $\left|v_{+}\right|$is the Jouguet velocity $v_{J}^{\text {det }}$, with $v_{J}^{\text {det }}>c_{+}$. For deflagrations we have $\left|v_{+}\right|<\left|v_{-}\right|$, and $\left|v_{+}\right|$has a maximum value $v_{J}^{\text {def }}<c_{+}$at the Jouguet point $\left|v_{-}\right|=c_{-}$. The hydrodynamical process is called weak if the velocities $v_{+}$and $v_{-}$are either both supersonic or both subsonic. Otherwise, the hydrodynamical process is called strong.

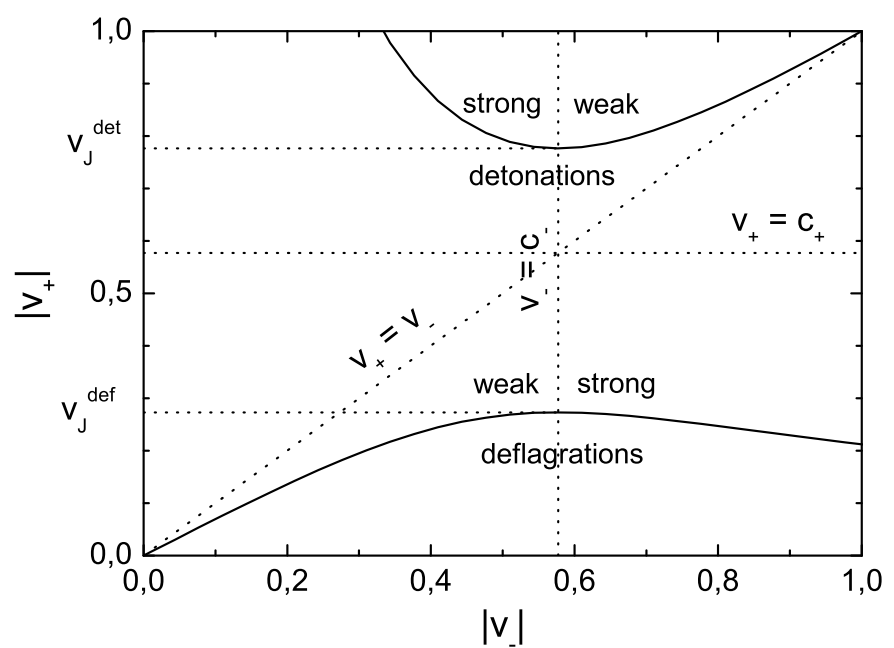

Figure 1: $v_{+}$vs $v_{-}$for the bag $\operatorname{EOS}\left(c_{+}=c_{-}=1 / \sqrt{3}\right)$, for $\alpha_{+}=0.1$. The upper branch corresponds to detonations and the lower to deflagrations.

We may also have discontinuities in the fluid profiles out of the bubble wall, which are called shock fronts. In the reference frame of these surfaces Eqs. (667) apply. The functions $w(T)$ and $p(T)$ are the same on both sides of the surface (since we have the same phase), but the temperature is discontinuous.

Away from the phase transition front, the field $\phi$ is constant and Eq. (1) becomes

$$
\begin{aligned}
\partial_{t}\left(w \gamma^{2}-p\right)+\partial_{x}\left(w \gamma^{2} v\right) & =0, \\
\partial_{t}\left(w \gamma^{2} v\right)+\partial_{x}\left[\left(w \gamma^{2} v^{2}+p\right)\right] & =0 .
\end{aligned}
$$

Since there is no characteristic distance scale in Eqs. (88,9), it is usual to assume the similarity condition [22], namely, that $w, p$ and $v$ depend only on $\xi=x / t$. Using the relation $p^{\prime}=c^{2} \rho^{\prime}$, where a prime indicates derivative with respect to $\xi$, we obtain the equation for the velocity profile (see e.g. [17])

$$
\left[\left(\frac{\xi-v}{1-\xi v}\right)^{2}-c^{2}\right] v^{\prime}=0 .
$$


Therefore we have either $v^{\prime}=0$, which gives constant solutions

$$
v(\xi)=\text { constant }
$$

or $(\xi-v) /(1-\xi v)= \pm c$, which gives two additional solutions. Of these, only the one corresponding to the + sign will be realized for the boundary conditions of our problem,

$$
v_{\mathrm{rar}}(\xi)=\frac{\xi-c}{1-\xi c}
$$

which corresponds to a rarefaction profile. The speed of sound $c$ in principle depends on $T$ and, thus, may depend on $\xi$. From Eqs. (8) we also obtain the equation for the enthalpy profile,

$$
\frac{w^{\prime}}{w}=\left(\frac{1}{c^{2}}+1\right) \frac{\xi-v}{1-\xi v} \gamma^{2} v^{\prime},
$$

which can be readily integrated to obtain the profiles of the thermodynamical variables. In particular, for $v=$ constant we have $T=$ constant.

The fluid velocity and temperature profiles are constructed with the solutions (11] 13), using the matching conditions (6-7) and appropriate boundary conditions. The usual boundary conditions consist of a vanishing fluid velocity far behind the moving wall (at the center of the bubble) and far in front of the wall, where information on the bubble has not arrived yet. The temperature far in front of the wall can be determined from the dynamics of the phase transition. We shall assume it is given by the nucleation temperature $T_{n}$ of the bubble. Three kinds of solutions are compatible with all these requirements (see [17] for details):

A detonation. The wall is supersonic and the fluid in front of it is unperturbed (see Fig. 2). Therefore, the fluid velocity in front of the wall vanishes, and we have $v_{w}=-v_{+}$, with $\left|v_{+}\right| \geq v_{J}^{\text {det }}$. It turns out that the fluid profile is only compatible with a weak detonation. Thus, the outgoing flow is also supersonic, $\left|v_{-}\right| \geq c_{-}$. Behind the wall, the fluid velocity is a constant between a certain point $\xi_{0}$ and $\xi_{w}=v_{w}$. At $\xi=\xi_{0}$ the fluid velocity matches the rarefaction solution (12), which vanishes at $\xi=c_{-}$. For $\xi<c_{-}$ we have $v=0$. As we shall see, the fluid profile behind the wall does not play a role in the determination of the detonation wall velocity.

A "traditional" deflagration. For this solution the fluid behind the wall is at rest, so $v_{w}=-v_{-}$. The deflagration could in principle be weak, Jouguet, or strong (however, the latter seems to be unstable [10, 11, 12]). The fluid velocity in front of the wall is a constant up to a shock front $\xi_{\text {sh }}$, which moves supersonically. Beyond the shock, the fluid is still unperturbed.

A supersonic deflagration. The hydrodynamical solution is a Jouguet deflagration, i.e., $\left|v_{+}\right|<\left|v_{-}\right|$and $\left|v_{-}\right|=c_{-}$. Hence, we have $\left|v_{+}\right|=v_{J}^{\text {def }}<c_{+}$. In this case, neither the fluid behind the wall nor in front of it is at rest, and the Jouguet condition $v_{-}=-c_{-}$ replaces the condition $v_{-}=-v_{w}$ of the traditional deflagration. Since the wall moves at the speed of sound with respect to the fluid behind it, and the fluid also moves (dragged by the wall), the wall velocity is always supersonic. The fluid profile behind the wall is 
given by the rarefaction solution (12) between $c_{-}$and $\xi_{w}$. In front of the wall, the fluid velocity is a constant up to the shock front.

Fixing the thermodynamical parameters, the latter solution exists for $c_{-} \leq v_{w} \leq v_{J}^{\text {det }}$. As the wall velocity of the supersonic deflagration approaches the limit $\xi_{w}=c_{-}$, the rarefaction wave disappears and this solution matches the traditional deflagration. As $\xi_{w}$ increases, the shock front and the phase-transition front become closer. In the limit $\xi_{w}=v_{J}^{\text {det }}$, the shock wave disappears and the solution matches the detonation.
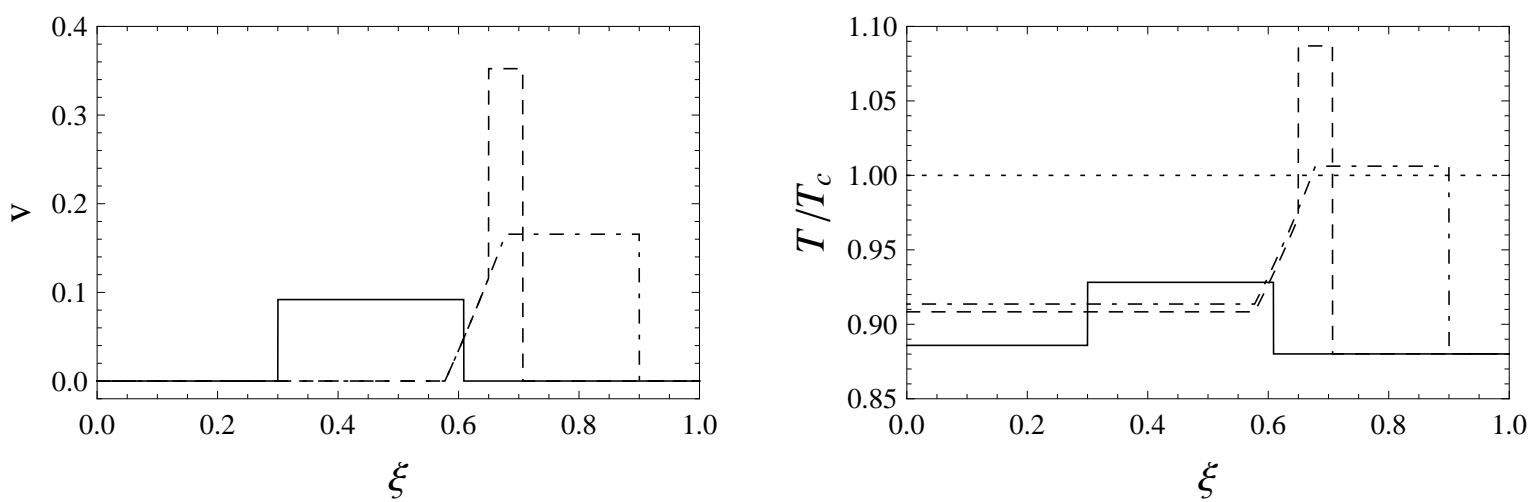

Figure 2: Some fluid profiles for the bag EOS with $\alpha_{n}=0.1$ and $\alpha_{c}=0.06\left(T_{n} / T_{c} \simeq 0.88\right)$. Solid lines correspond to a weak deflagration with $v_{w}=0.3$, dashed lines to a Jouguet deflagration with $v_{w}=0.65$, and dashed-dotted lines to a weak detonation with $v_{w}=0.9$. Left: the fluid velocity. Right: the fluid temperature. The dotted line indicates the critical temperature.

\subsection{Microphysics}

In order to obtain analytical results for the wall velocity, we shall implement the thin wall approximation in the friction equation (5). Following Ref. [14], we multiply Eq. (5) by $d \phi / d x$, integrate across the wall, and use $(\partial \mathcal{F} / \partial \phi)(d \phi / d x)=d \mathcal{F} / d x-(\partial \mathcal{F} / \partial T)(d T / d x)$ [10]. We obtain

$$
p_{+}-p_{-}-\int\left(-\frac{\partial \mathcal{F}}{\partial T}\right) d T-\int \tilde{\eta} T f(\phi / T)\left(\phi^{\prime}\right)^{2} v \gamma d x=0,
$$

where $\phi^{\prime} \equiv d \phi / d x$. We will make approximations for the integrals in Eq. (14), so that we can express the result in terms of the values of the variables outside the wall.

First, we write the friction term as

$$
-\int \tilde{\eta} T f(\phi / T)\left(\phi^{\prime}\right)^{2} v \gamma d x \equiv \eta \frac{\left|v_{+}\right| \gamma_{+}+\left|v_{-}\right| \gamma_{-}}{2}
$$

(notice that the fluid velocity $v$ in the rest frame of the wall is negative). The friction coefficient $\eta$ is usually approximated by its value in the limit $T_{+}=T_{-}=T_{n}$, i.e., when 
the effects of hydrodynamics are neglected and only microphysics is considered. This is the limit in which the fluid is left unperturbed by the wall, and is valid for very weak solutions, for which $v_{+}=v_{-}=-v_{w}$. According to Eqs. (14-15), the wall velocity is given in this limit by

$$
\left.v_{w} \gamma_{w}\right|_{\text {micro }}=\frac{p_{-}\left(T_{n}\right)-p_{+}\left(T_{n}\right)}{\eta}
$$

with $\eta$ given by

$$
\eta=\tilde{\eta} T \int f(\phi / T)\left(\phi^{\prime}\right)^{2} d x
$$

We shall consider $\eta$ as a constant in Eq. (15), which can be calculated for a specific model using the approximation (17). Similarly, for the first integral in Eq. (14) we shall use a linear approximation for the entropy density inside the wall, which gives

$$
\int\left(-\frac{\partial \mathcal{F}}{\partial T}\right) d T \simeq \frac{\left(s_{+}+s_{-}\right)\left(T_{+}-T_{-}\right)}{2}
$$

With these approximations, Eq. (14) becomes [14]

$$
p_{+}-p_{-}-\frac{s_{+}+s_{-}}{2}\left(T_{+}-T_{-}\right)+\frac{1}{2} \eta\left(\left|v_{+}\right| \gamma_{+}+\left|v_{-}\right| \gamma_{-}\right)=0 .
$$

This expression is valid for any specific choice of the effective potential, since the information on the EOS is encoded in the variables $p$ and $s$. Furthermore, considering a different phenomenological damping only amounts to modifying the function of $v_{+}$and $v_{-}$in the last term and is straightforward, as we shall see in section 6 with an example. Therefore, Eq. (19) is readily applicable to a large variety of models.

The approximation (17) neglects the variation of the fluid velocity inside the wall, since it is obtained from Eq. (15) by replacing $v \gamma$ with its mean value. We expect this approximation to be better for weak solutions. Jouguet and strong processes cause larger perturbations of the fluid, and will give larger deviations. Indeed, as can be seen in Fig. 1, for weak solutions the difference between $v_{+}$and $v_{-}$is maximal at the Jouguet point. Similarly, Eq. (18) approximates the entropy density inside the wall by the mean value $\left(s_{+}+s_{-}\right) / 2$. We expect this approximation to be good for weaker solutions and to fail perhaps for Jouguet or stronger solutions, for which the difference between + and variables can be large. As we shall see, since only weak or, at most, Jouguet processes are actually realized in the phase transition, the strongest deviations from the correct results will indeed occur for velocities which are close to the Jouguet deflagration or detonation.

The friction force was calculated for some specific models for the case of the electroweak phase transition [21]. In the thin wall approximation, the wall profile can be estimated by neglecting the temperature variation and the last term in Eq. (5). Thus, we have $d \phi / d x=-\sqrt{2 \Delta \mathcal{F}(\phi, T)}$, with

$$
\Delta \mathcal{F}(\phi, T) \equiv \mathcal{F}(\phi, T)-\mathcal{F}(0, T)
$$


and Eq. (17) gives

$$
\eta \simeq \tilde{\eta} T \int f(\phi / T) \sqrt{2 \Delta \mathcal{F}(\phi, T)} d \phi .
$$

Roughly, we have $\eta \approx \tilde{\eta} T \sigma$, where $\sigma=\int \phi^{2} d x$ is the surface tension of the bubble wall. This corresponds to setting $f(\phi / T)=1$. The function $f$, though, can make a quantitative difference (see, e.g., [14, 20]). However, since we are not going to consider any particular model but rather take $\tilde{\eta}$ as a free parameter, a different $f$ in Eq. (21) only amounts to a redefinition of $\tilde{\eta}$.

\section{The bag EOS}

Equations (6), (7) and (19) can be solved for the wall velocity once the equation of state of the system is known. It is convenient to use as an approximation the bag EOS,

$$
\mathcal{F}_{+}(T)=-a_{+} T^{4} / 3+\varepsilon, \quad \mathcal{F}_{-}(T)=-a_{-} T^{4} / 3 .
$$

This simplification allows to find analytical expressions for the solutions. In this model the latent heat is given by $L=4 \varepsilon$ and the speed of sound is a constant $c=1 / \sqrt{3}$ in both phases. It is customary to express the results as functions of the variable $\alpha \equiv \varepsilon /\left(a_{+} T^{4}\right)$. As discussed in Refs. [14, 20], for applications it is convenient to use $L$ instead of $\varepsilon$. Therefore, we define the parameters

$$
\alpha_{c}=\frac{L}{4 a_{+} T_{c}^{4}}, \quad \alpha_{+}=\frac{L}{4 a_{+} T_{+}^{4}}, \quad \alpha_{n}=\frac{L}{4 a_{+} T_{n}^{4}} .
$$

For the bag EOS Eqs. (6) and (7) give

$$
v_{+}=\frac{\frac{1}{6 v_{-}}+\frac{v_{-}}{2} \pm \sqrt{\left(\frac{1}{6 v_{-}}+\frac{v_{-}}{2}\right)^{2}+\alpha_{+}^{2}+\frac{2}{3} \alpha_{+}-\frac{1}{3}}}{1+\alpha_{+}},
$$

which is plotted in Fig. 1 for $\alpha_{+}=0.1$. The plus sign corresponds to detonations and the minus sign to deflagrations. The friction equation (19) can also be expressed in terms of $v_{+}, v_{-}$, and $\alpha_{+}$,

$$
\frac{4 v_{+} v_{-} \alpha_{+}}{1-3 v_{+} v_{-}}-\frac{2}{3}\left(1+\frac{s_{-}}{s_{+}}\right)\left(1-\frac{T_{-}}{T_{+}}\right)+\frac{2 \alpha_{+} \eta}{L}\left(\left|v_{+}\right| \gamma_{+}+\left|v_{-}\right| \gamma_{-}\right)=0
$$

with

$$
\frac{s_{-}}{s_{+}}=\frac{a_{-}}{a_{+}}\left(\frac{T_{-}}{T_{+}}\right)^{3} \text { and } \frac{T_{-}}{T_{+}}=\left[\frac{a_{+}}{a_{-}}\left(1-\alpha_{+} \frac{1+v_{+} v_{-}}{1 / 3-v_{+} v_{-}}\right)\right]^{1 / 4} .
$$

The latter expressions depend on the ratio $a_{-} / a_{+}$, which introduces a dependence on the parameter $\alpha_{c}$,

$$
a_{-} / a_{+}=1-3 \alpha_{c} .
$$


From Eqs. (24-25) we can find the velocities $v_{+}$and $v_{-}$as functions of $\eta / L, \alpha_{+}$and $\alpha_{c}$. However, the variable $\alpha_{+}$can be eliminated, since the temperature $T_{+}$in front of the wall is a function of the nucleation temperature $T_{n}$. The relation between $\alpha_{+}$and $\alpha_{n}$ depends on the type of hydrodynamic solution. For detonations the temperature $T_{+}$is just given by $T_{+}=T_{n}$, and hence $\alpha_{+}=\alpha_{n}$. For deflagrations, $T_{+}$is related to $T_{n}$ through the matching conditions at the shock discontinuity,

$$
v_{1} v_{2}=\frac{1}{3}, \quad \frac{v_{1}}{v_{2}}=\frac{3 T_{n}^{4}+T_{+}^{4}}{3 T_{+}^{4}+T_{n}^{4}},
$$

where $v_{1}$ is the velocity of the outgoing flow in the reference frame of the shock, and $v_{2}$ that of the incoming flow. In the rest frame of the bubble center, the fluid velocity in front of the shock vanishes (see Fig. 2). Hence, the velocity of the shock is given by $v_{\mathrm{sh}}=-v_{2}$. In the shock-wave region the fluid velocity is a constant and, thus, can be obtained either from the velocity $v_{+}$or from the velocity $v_{1}$. This gives the equation

$$
\frac{v_{w}-\left|v_{+}\right|}{1-\left|v_{+}\right| v_{w}}=\frac{\sqrt{3}\left(\alpha_{n}-\alpha_{+}\right)}{\sqrt{\left(3 \alpha_{n}+\alpha_{+}\right)\left(3 \alpha_{+}+\alpha_{n}\right)}} .
$$

Fixing the friction and the critical temperature, we can use the above equations to obtain $v_{w}$ as a function of $\alpha_{n}$ as follows. For detonations we have $v_{+}=-v_{w}$. We can eliminate $v_{-}$from Eq. (24), and then obtain $v_{w}$ from Eq. (25) as a function of $\alpha_{+}=\alpha_{n}$. For traditional deflagrations, we have $v_{-}=-v_{w}$, so we can eliminate $v_{+}$using Eq. (24) and obtain $v_{w}$ from Eq. (25) as a function of $\alpha_{+}$. Then, Eq. (29), together with Eq. (24), can be used to obtain $\alpha_{+}$as a function of $\alpha_{n}$ and $v_{w}$. For Jouguet deflagrations, we have $v_{-}=-1 / \sqrt{3}$ fixed, so Eq. (24) alone gives $v_{+}$as a function of $\alpha_{+}$, i.e., $v_{+}=v_{J}^{\text {def }}\left(\alpha_{+}\right)$. Therefore, Eq. (25) gives already the value of $\alpha_{+}$(as a function of the parameters $\eta$ and $\alpha_{c}$ ). In this case, Eq. (29) can be used to obtain the wall velocity as a function of $\alpha_{+}$and $\alpha_{n}$.

\section{General structure of the solutions}

Solving Eqs. (24-29) just amounts to finding the roots of algebraic equations, thus avoiding time-consuming numerical calculations. This is valuable when considering the development of a phase transition. However, in the way from Eqs. (3,5) to Eqs. (24,29) we have made several approximations. Therefore, it is important to determine the range of validity of these results.

It is useful to consider the structure of the stationary states in the $T_{+} T_{-}$-plane. Following [11, we consider, on the one hand, the solutions which satisfy the energy-momentum conservation and the friction equation, i.e., Eqs. (35 (5), but for which the boundary conditions have not been imposed. For the bag EOS and our analytical approximations, this amounts to considering Eq. (25), with $v_{+}$and $v_{-}$expressed in terms of $T_{+}$and $T_{-}$,

$$
v_{-}^{2}=\frac{\left(1 / 3-\alpha_{+}-r / 3\right)\left(1+\alpha_{+}+r / 3\right)}{\left(1+\alpha_{+}-r\right)\left(r+1 / 3-\alpha_{+}\right)}
$$




$$
v_{+}^{2}=\frac{\left(1 / 3-\alpha_{+}-r / 3\right)\left(r-\alpha_{+}+1 / 3\right)}{\left(1+\alpha_{+}-r\right)\left(1+r / 3+\alpha_{+}\right)},
$$

with

$$
r \equiv \frac{a_{-}}{a_{+}} \frac{T_{-}^{4}}{T_{+}^{4}}
$$

This gives curves of $\eta=$ constant in the $T_{+} T_{-}$-plane (see Fig. 3). On the other hand, we consider the solutions which satisfy the energy-momentum conservation equations, Eqs. (3-4), and the boundary conditions, but for which the friction equation (5) has not been imposed. This gives curves of fixed $T_{n}$ in the $T_{+} T_{-}$-plane. For detonations, these are curves of constant $T_{+}=T_{n}$. For traditional deflagrations, the curves are given by Eq. (29) with $v_{w}=\left|v_{-}\right|$, and with $v_{ \pm}$given by Eqs. (30-32). Jouguet solutions are given by the condition $v_{-}^{2}=1 / 3$. The values of $T_{+}$and $T_{-}$are constrained by the conditions $0<v_{ \pm}^{2}<1$ and by the production of entropy at the phase transition front, $s_{-}\left|v_{-}\right| \gamma_{-} \geq s_{+}\left|v_{+}\right| \gamma_{+}$.

The result is shown in Fig. 3. The dark grey region is forbidden by kinematics and by the condition of non-negative entropy production. There are two allowed regions. The upper one corresponds to deflagrations and the lower one to detonations. Weak deflagrations (white zone) are separated from strong deflagrations (light grey zone) by a dashed line which indicates Jouguet processes. The deflagration region is delimited by the line of zero entropy production (curved part of the boundary) and by the line of $v_{-}=0$ (straight part of the boundary). The lower white region, corresponding to weak detonations, is delimited by the Jouguet condition (dashed line), the zero entropy-production condition (curved boundary), and the condition $v_{+}=1$ (upper straight boundary). Solid lines represent the solutions of constant $\eta$. Dotted lines represent the solutions for a given temperature $T_{n}$ for traditional deflagrations and for detonations. For detonations, the dotted curves are horizontal lines due to the boundary condition $T_{+}=T_{n}$. For the traditional deflagrations, the curves are almost vertical lines, indicating that, although the temperature $T_{+}$can be quite higher than $T_{n}$ due to reheating in front of the wall, the temperature $T_{-}$inside the bubble is very close to $T_{n}$ [11. If we plotted the dotted curves also for supersonic Jouguet deflagrations, they would lie on the dashed line separating weak and strong deflagrations.

The possible stationary states correspond to the intersections of solid and dotted curves in Fig. 3. Thus, we see that for some values of the parameters (e.g., for $T_{n} / T_{c}=0.95$, $\tilde{\eta}=0.04)$ we will have multiple solutions for the wall velocity [10, 11, 12]. Notice also that solid lines in the deflagration region approach asymptotically the kinematic boundary corresponding to $v_{-}=0$. As a consequence, for large enough $\tilde{\eta}$ or for $T_{n}$ close enough to $T_{c}$, there will always be weak deflagration solutions. Similarly, solid lines in the detonation region approach asymptotically the boundary of $v_{+}=1$. Therefore, there will always be detonations for small enough friction or strong enough supercooling. For intermediate values of $\tilde{\eta}$ and $T_{n} / T_{c}$, it may happen that neither weak deflagrations nor detonations exist, as e.g. for the case $\tilde{\eta}=0.1, T_{n} / T_{c}=0.89$ (whereas for $T_{n} / T_{c}=0.86$ we have a detonation and for $T_{n} / T_{c}=0.92$ we have a weak deflagration). Generally, in such a case there will exist supersonic Jouguet deflagrations. 


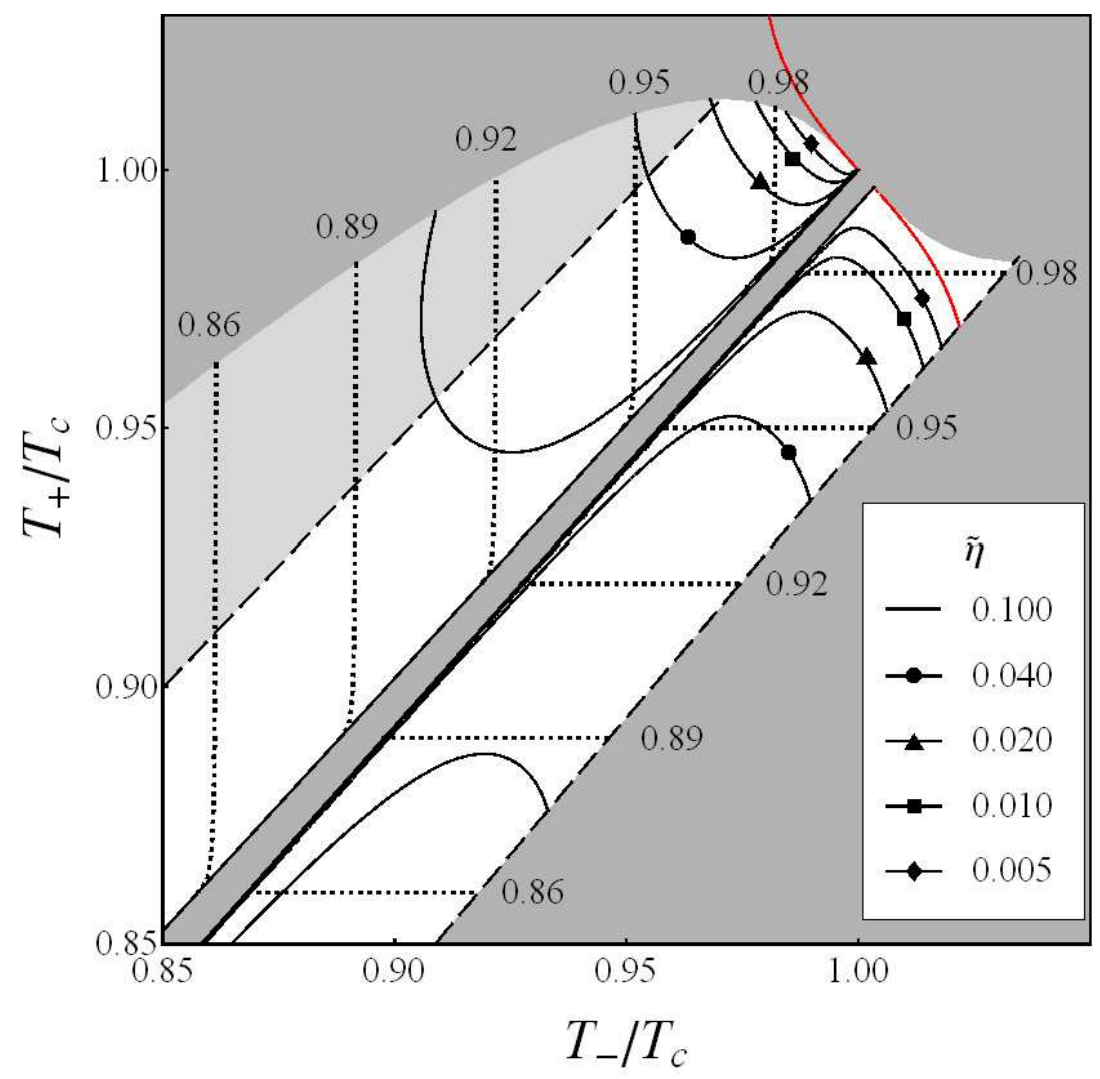

Figure 3: The solutions in the $T_{+} T_{-}$-plane for $a_{+}=\left(\pi^{2} / 90\right) g_{*}$ with $g_{*}=51.25, L=$ $0.1 T_{c}^{4}$, and $\eta=\tilde{\eta} T \sigma$, with $\sigma=0.1 T_{c}^{3}$. The region with the dark shade is forbidden by kinematics or by the non-negativity of entropy production. The upper allowed region corresponds to deflagrations, and the lower one to detonations. The Jouguet processes are indicated by dashed lines, and the region with a lighter shade corresponds to strong deflagrations. Dotted lines correspond to fixed values of $T_{n} / T_{c}=0.86,0.89,0.92,0.95$ and 0.98 as indicated, and solid lines to $\tilde{\eta}$ fixed (see explanation in the text). The red line corresponds to $\tilde{\eta}=0$.

In Refs. [10, 11, 12], the differential equations for $\phi(x), T(x)$, and $v(x)$ were solved numerically using a grid. Furthermore, a $\phi$-dependent, quartic potential was considered as an approximation for the free energy. Comparing the results of those numerical computations with our results, we can test to what extent the use of the bag EOS, the thin wall limit and the approximations (15) 19) are valid. Thus, in Fig. 3 we have considered "QCD-type" parameters used in Refs. [10, 11, 12] and we have set $f(\phi / T)=1$ in Eq. (21), which gives a friction of the form $\eta=\tilde{\eta} T \sigma$. The value of $a_{+}$is given by $a_{+}=\left(\pi^{2} / 90\right) g_{*}$, where the number of effective degrees of freedom in the high-temperature phase is $g_{*}=51.25$. The latent heat is given by $L=0.1 T_{c}^{4}$, and the surface tension is $\sigma=0.1 T_{c}^{3}$. The set of values for the friction and for the nucleation temperature are the same as in Fig. 1 of Ref. [11]. 
The dotted lines, as well as the limits of the allowed region, are qualitatively identical and quantitatively quite close to those of Ref. [11]. This indicates that the bag model is a good approximation for the EOS, at least for QCD-type parameters. In the white region (i.e., that of weak solutions) the solid curves are quantitatively similar to those of Ref. [11]. Qualitatively, the curves deviate from those of Ref. [11] as they approach the dashed lines (i.e., as the solutions approach a Jouguet process). This is most apparent for $\tilde{\eta} \rightarrow 0$. In the plot of Ref. [11], for small friction the solid curves are parallel to the border of the allowed region (in the top-right corner of our Fig. 33). This is because the entropy production is related to gradients of $\phi$ through the friction parameter $\tilde{\eta}$. Indeed, combining Eqs. (35), one obtains

$$
T \frac{d}{d x}\left(-\frac{\partial \mathcal{F}}{\partial T} \gamma v\right)=\tilde{\eta} T(\gamma v)^{2} f(\phi / T) \phi^{\prime}(x)^{2},
$$

which yields

$$
s_{-}\left|v_{-}\right| \gamma_{-}-s_{+}\left|v_{+}\right| \gamma_{+}=\tilde{\eta} \int(\gamma v)^{2} f(\phi / T) \phi^{\prime}(x)^{2} d x .
$$

As a consequence, the solid curve for $\tilde{\eta}=0$ should coincide with the limit between the white and grey regions, corresponding to zero entropy production. Instead of that, in our case this curve (red line) enters the allowed region for detonations and enters the forbidden region for deflagrations. This is an indication of the break-down of the approximation for the integral of the entropy density across the wall, Eq. (18), as weak solutions approach a Jouguet process (see the discussion in subsection 2.2). However, as we shall see in the next section, in most cases our analytical approximations give values of the wall velocity which are qualitatively and quantitatively good, even for solutions near the Jouguet point.

In Ref. [11, the solid lines in the $T_{+} T_{-}$-plane do not penetrate the strong deflagration region but stop at the Jouguet line. This means that the numerical code did not find any strong deflagrations. As explained in Ref. [11], this is due to the fact that no matter how well one tries to guess the correct solution, the guess does not relax to a strong deflagration but rather changes considerably to form a different type of solution. This is an indication that strong deflagrations are unstable. In Fig. 3 it is seen that we find strong deflagration solutions. We believe this is not a shortcoming of our approximations, but rather due to the fact that analytical equations allow to find any solution, even the unstable ones. In this region, though, the departure of our solid lines from the actual curves is probably large. In any case, being unstable, strong deflagrations are of little interest.

\section{The wall velocity}

Let us consider now the wall velocity as a function of the parameters. As can be seen in Fig. 4, weak traditional deflagrations (red curves) always exist for large friction $(\tilde{\eta} \gtrsim 1)$ or little supercooling $\left(\alpha_{n} \approx \alpha_{c}\right)$. For lower values of the friction or larger amounts of supercooling, the traditional deflagrations surpass the speed of sound, becoming strong 
deflagrations. Shortly after crossing the sound barrier, though, the red curves end due to the condition of non-negative entropy production. At this point we always have supersonic Jouguet deflagrations (black lines), which match the traditional deflagrations at $v_{w}=c$. For small enough friction or strong enough supercooling we always have detonations (blue lines).
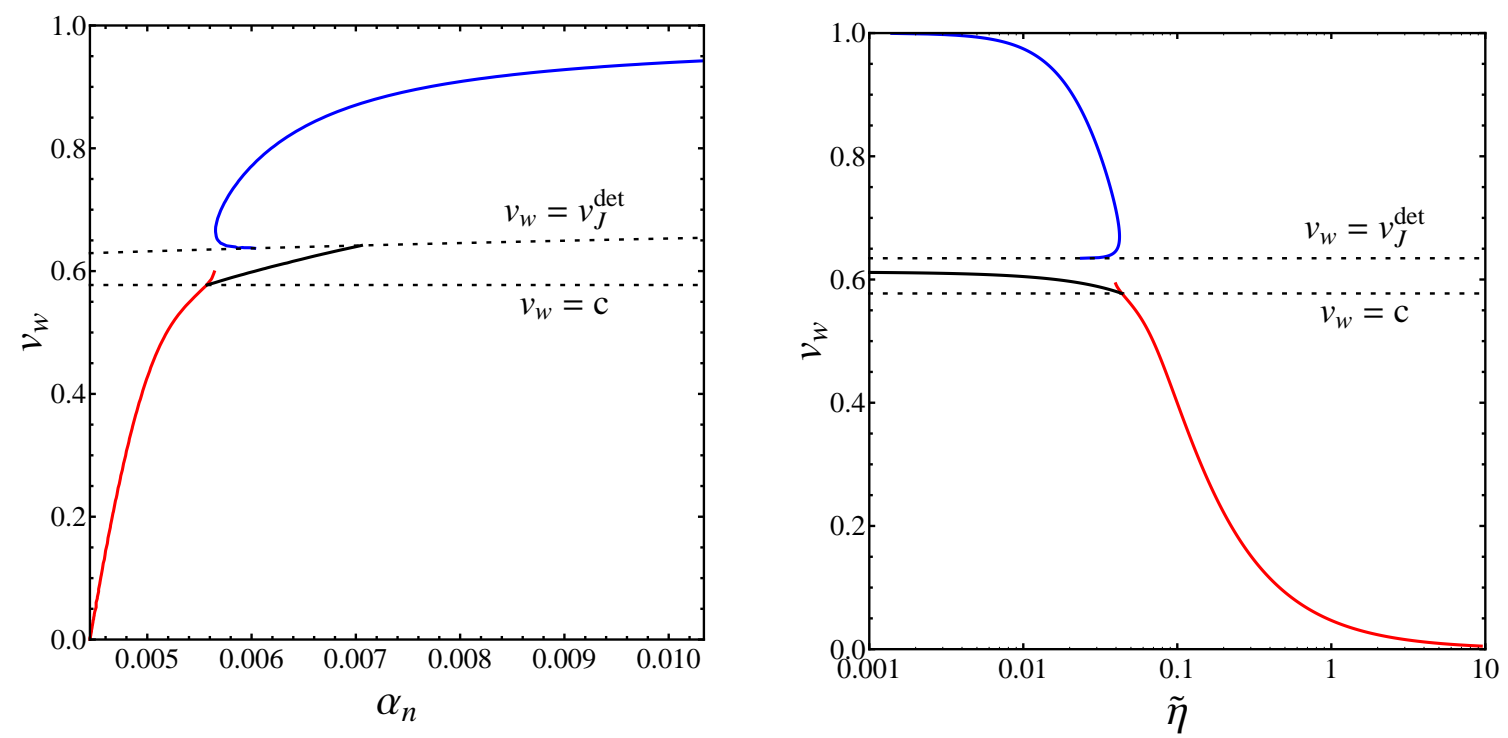

Figure 4: The wall velocity for $g_{*}=51.25, L=0.1 T_{c}^{4}$, and $\eta=\tilde{\eta} T \sigma$, with $\sigma=0.1 T_{c}^{3}$. Detonations are plotted in blue, Jouguet deflagrations are plotted in black, and traditional deflagrations are in red. The dotted lines correspond to the sound and Jouguet velocities. Left: the wall velocity as a function of $\alpha_{n}$, for $\tilde{\eta}=0.05$. The range of $\alpha_{n}$ correspond to values of the temperature between $T_{n}=T_{c}$ and $T_{n} \simeq 0.8 T_{c}$. Thus, the $\alpha_{n}$-axis begins at the value $\alpha_{n}=\alpha_{c} \simeq 4.45 \times 10^{-3}$. Right: the wall velocity as a function of the friction, for $T_{n}=0.95 T_{c}\left(\alpha_{n} \simeq 5.46 \times 10^{-3}\right)$.

Notice that, for some values of $\eta$ and $\alpha_{n}$, there are more than one stationary state. This can be seen already in Fig. 3 , since the solid and dotted curves intersect at several points. In Fig. 4 we show all the solutions for a given friction and supercooling. In some ranges of the parameters we have, for instance, a deflagration and a detonation, or a deflagration and two detonations. This is in agreement with the results of Refs. [10, 11, 12]. We may also have two traditional deflagration solutions for a given set of parameters, as shown in the left panel of Fig. 5. One of these solutions is a strong deflagration.

One could expect that it will be always possible to choose the solution in such a way that, as the parameters are varied, one can go continuously from a subsonic, weak deflagration to a supersonic, Jouguet deflagration and then to a weak detonation. As seen in Figs. 4 and 5, one can indeed change continuously from a weak deflagration to a supersonic Jouguet deflagration. However, the Jouguet deflagration velocity does not match the detonation velocity. The continuity of the deflagration solutions is a consequence of 

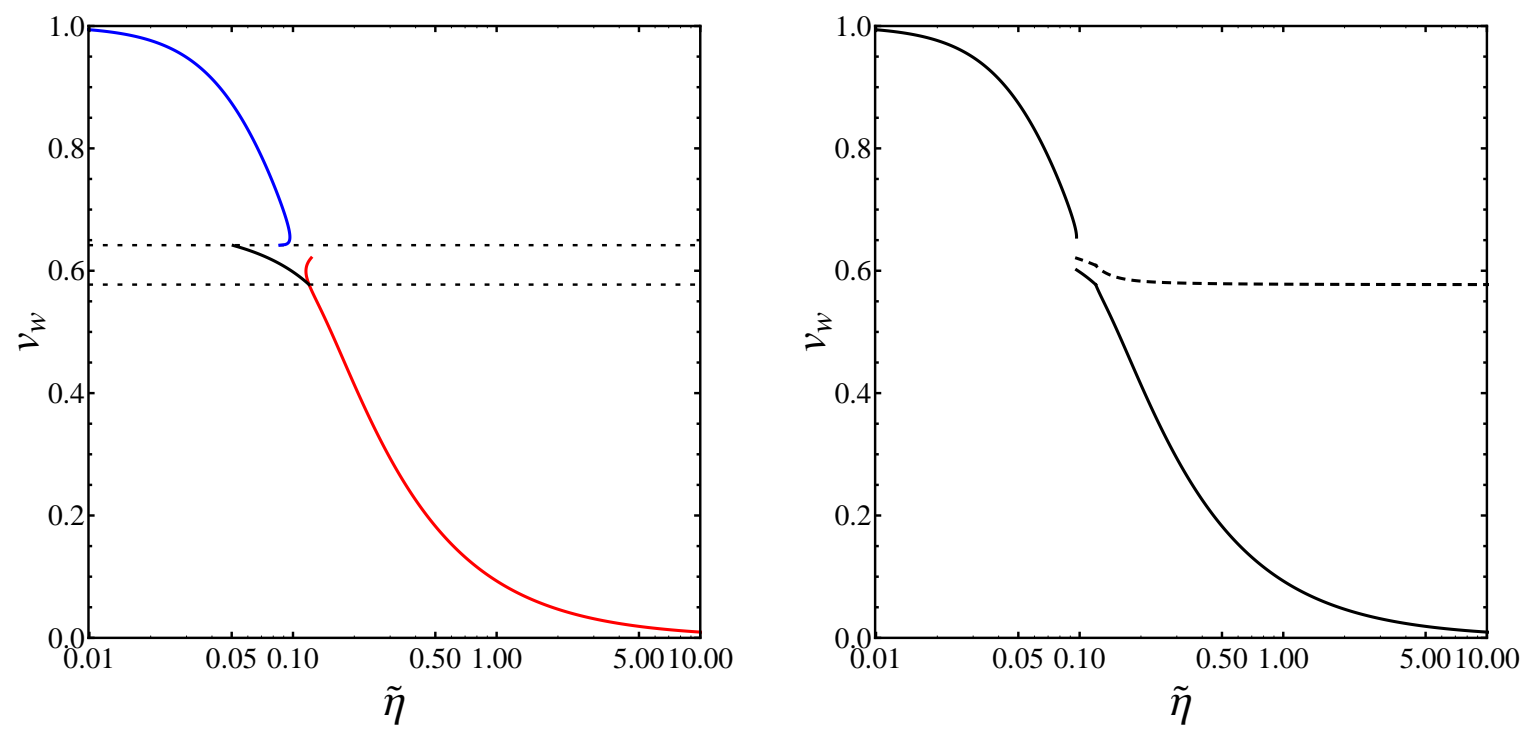

Figure 5: The same as in Fig. 4 (right), but for $T_{n}=0.891 T_{c}$. In the right panel only one of the multiple solutions has been chosen. The dashed line corresponds to the velocity of the shock front.

the continuity of the profiles. Consider the supersonic Jouguet deflagration. As the velocity approaches the speed of sound $c$, the rarefaction wave vanishes continuously. Both the height and the width of the rarefaction vanish, matching continuously the profile of the traditional deflagration. In contrast, as the velocity approaches the Jouguet detonation velocity $v_{J}^{\text {det }}$, the shock wave becomes thinner and higher. Thus, the fluid velocity in front of the wall is maximal for a Jouguet deflagration at $v_{w}=v_{J}^{\operatorname{det}}$ (while the width of the shock wave vanishes). On the contrary, for a detonation the fluid velocity always vanishes in front of the wall. Therefore, the fluid velocity is discontinuous as the solution changes from a Jouguet deflagration to a detonation. This originates a jump in the parameters $\alpha_{n}$ and $\tilde{\eta}$ as functions of $v_{w}$, as can be seen in Figs. 4 and 5 ,

Leaving aside the jump of the parameters at $v_{w}=v_{J}^{\text {det }}$, one would expect that the supersonic Jouguet deflagration will always fill the velocity gap between weak deflagrations and weak detonations (as, e.g., in the left panels of Figs. 4 and 5). A fluid profile does exist for any value of the wall velocity in the range $c \leq v_{w} \leq v_{J}^{\text {det }}$. The Jouguet deflagration reaches the value $v_{w}=c$ for the same values of parameters as the weak deflagration, whereas the value $v_{w}=v_{J}^{\text {det }}$ is reached for a lower friction or a stronger supercooling than the detonation. This behavior can be explained by the fact that, for deflagrations, the compression wave which propagates in front of the wall and reheats the fluid, causes a friction effect [14] which adds to the microphysics. Technically, the friction effect for the deflagration arises as a consequence of the relation (29) between the nucleation temperature $T_{n}$ and the reheated value $T_{+}$. For the detonation, instead, we have $T_{+}=T_{n}$. This effect is easily estimated for weak solutions [14]. In the small supercooling limit (i.e., for 
weak deflagrations) we have $v_{w}=\Delta p\left(T_{n}\right) / \eta_{\text {eff }}$, where $\Delta p$ is the pressure difference $p_{-}-p_{+}$ and the effective friction is given by $\eta_{\mathrm{eff}}=\left(w_{-} / w_{+}\right)\left[\eta+L^{2} /\left(\sqrt{3} w_{-}\right)\right]$, which doesn't vanish for $\eta=0$. In the ultrarelativistic limit, in contrast, we have weak detonations with $v_{w}=1-\delta$, where $\delta$ is proportional to $\eta^{2}$ and vanishes for vanishing friction. For deflagrations with high wall velocities $\left(v_{w} \gtrsim c\right)$ the fluid velocity and temperature profiles have a sharp peak in front of the wall, so the friction effect can be considerably large. Notice that, in the right panel of Fig. 4, the value $v_{J}^{\text {det }}$ is never reached by Jouguet deflagrations, even in the limit $\tilde{\eta} \rightarrow 0$. Thus, we see that in some cases the effective friction can prevent the supersonic deflagration to reach the velocity of the Jouguet detonation.

Even in the cases in which the Jouguet deflagrations fill the whole range between $c$ and $v_{J}^{\mathrm{det}}$, only one of the multiple solutions will be realized. As a consequence, there will always be a gap in the velocity, as shown in Fig. 5 (right panel). It is important to determine which of the possible solutions will be realized in the phase transition as a stationary state. In Refs. [10, 11, 12, the evolution from a given initial configuration of the scalar field and the fluid variables (i.e., from an initial "bubble wall") was studied by numerically solving the time dependent partial differential equations. Thus, their dynamical code selected one of the possible final states. It was observed that the strong deflagrations, as well as the branch of detonations which are closer to the Jouguet point, are not realized in the evolution of the bubble wall. Moreover, even if given as initial conditions, these solutions transform into one of the other solutions, suggesting an instability [11. Furthermore, as seen in Figs. 4 and 5 , these solutions have a non-physical behavior as functions of the parameters [14]. Therefore, they must be discarded. In contrast, weak deflagrations, Jouguet deflagrations, and the branch of weaker detonations are in general stable. For high friction or low supercooling we can only have weak deflagrations. As the parameters are varied and the weak deflagration reaches the velocity $v_{w}=c$, we must change to a Jouguet deflagration. On the other hand, for $v_{w} \gtrsim c$ we will have to choose between a deflagration and a detonation. Both are stable and both are reachable from different initial conditions in the dynamical code of Ref. [11]. However, in the normal evolution of a bubble wall, the solution which is realized is the detonation 1 . Accordingly, we must always choose the detonation if possible; otherwise, the Jouguet deflagration; otherwise, the weak deflagration. The result is shown in the right panel of Fig. 5, where we reproduced the plot of the left panel, keeping only the selected solutions.

Qualitatively, our results agree with the numerical solutions, even for spherical bubbles (cf. Fig. 3 of Ref. [12]). The parameters we used correspond to Fig. 13 of Ref. [10]. There, the friction parameter is $\Gamma=1 / \tilde{\eta}$. For a better comparison we plot the velocity as a function of $\Gamma$ in Fig. 6, For the range of friction considered in that figure, we have a difference of at most a $5 \%$ for weak deflagrations and less for detonations. For the weakest solutions the difference vanishes, as expected. Moreover, for the strongest solutions, i.e., those around the Jouguet point, the agreement is quite good, as the jump

\footnotetext{
${ }^{1}$ Interestingly, the solutions which are not realized are those which are closer to the speed of sound and have a sharp peak in temperature. Thus, the dynamical evolution selects the weaker stationary solution, i.e., the weak detonation. For the fastest deflagrations that are realized, the bubble wall first goes into a detonation configuration before settling into a deflagration [12].
} 
in $v_{w}$ is approximately at the same place, $\Gamma \approx 10$.

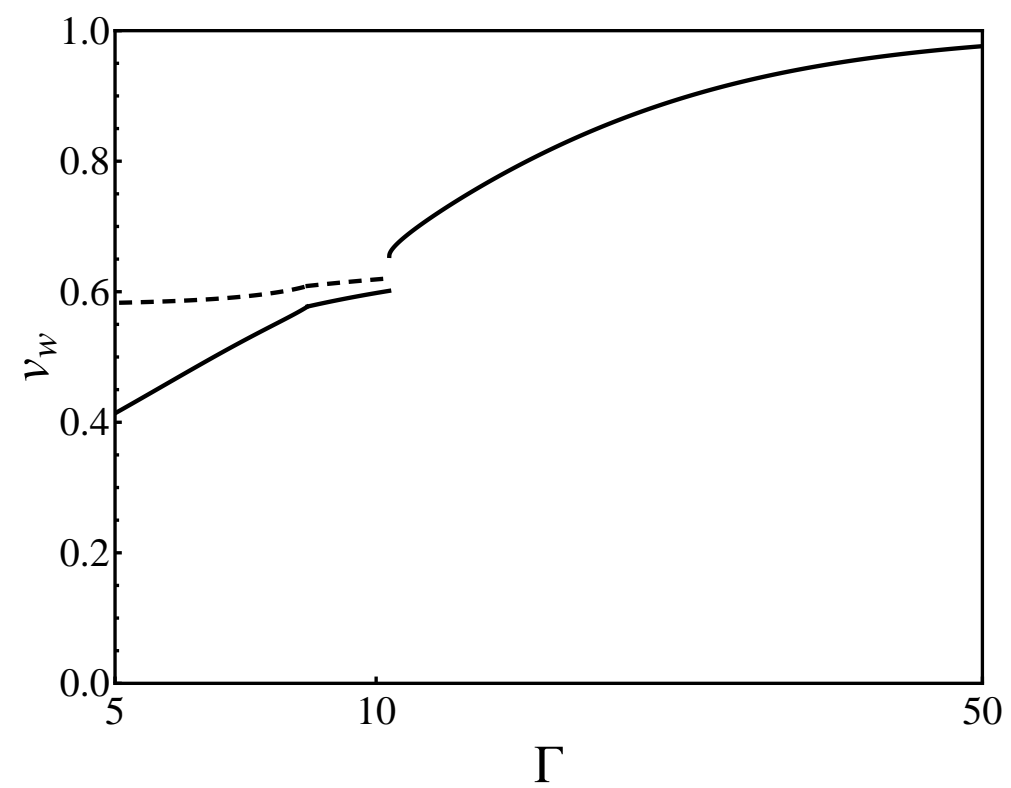

Figure 6: The wall (solid) and shock (dashed) velocities as functions of $\Gamma=1 / \tilde{\eta}$. The values of the parameters are as in Fig. 5 .

One expects that for any set of values of the parameters there will exist at least one solution. Consider a fixed value of $T_{n}$. For large friction there should always be weak deflagrations and for small friction there should always be weak detonations. In the intermediate range there should exist Jouguet deflagrations. Although this is in general true, we find some exceptions in extreme cases. For instance, for a large amount of supercooling, $T_{n} / T_{c}=0.6$ (which is out of the range of Fig. 3), we find that, as we decrease the friction, the Jouguet deflagration velocity reaches the value of the Jouguet detonation before the detonation solution appears (see Fig. 7, left panel). As a consequence, there is a range of values of $\tilde{\eta}$ for which there is no solution. For even stronger phase transitions, we find that there may be no solutions for large values of the friction. This behavior was observed also in Ref. [16]. This is shown in the right panel of Fig. 7, where we considered values of the parameters similar to those used in Ref. [16]. For larger amounts of supercooling, there may be no subsonic deflagrations at all, whereas supersonic deflagrations cease to exist at some maximum friction.

We have checked that, for physical models, the parameters (particularly the amount of supercooling) hardly fall into the case of Fig. 7. In particular, we considered the electroweak phase transition for several extensions of the Standard Model [9]. The situation of the right panel never arose, and that of the left panel arose only in a few limiting cases which are quite unlikely. For instance, extra scalars with very strong couplings with the Higgs field may yield an exceedingly strongly first-order phase transition. Only for some particular sets of parameters, and for the highest values of the couplings of the extra bosons, we found the situation of no stationary solution. Such couplings are ex- 

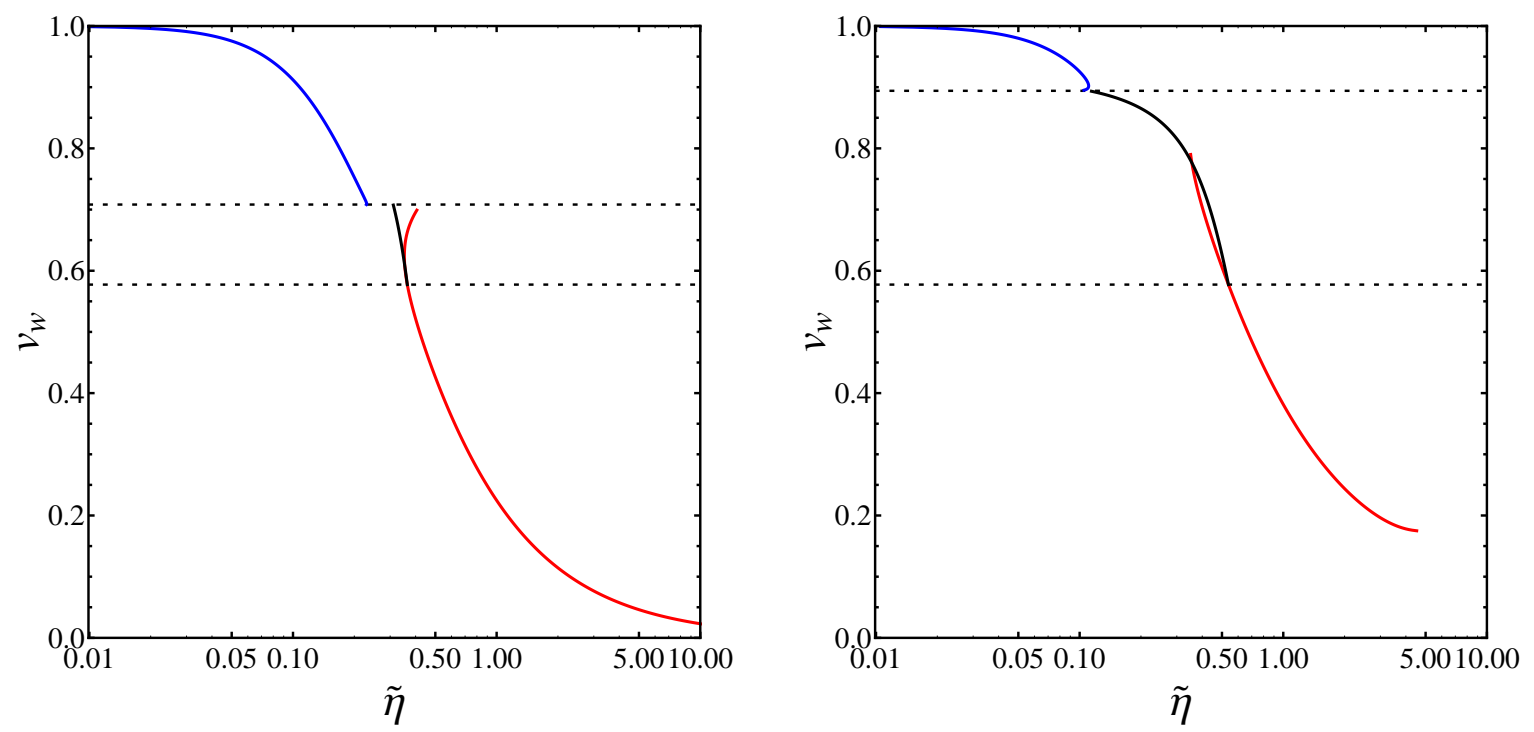

Figure 7: The wall velocity as a function of $\tilde{\eta}$ for $\alpha_{c}=4.45 \times 10^{-3}$ and $T_{n} / T_{c}=0.6$ (left panel), and for $\alpha_{c}=0.05\left(a_{-} / a_{+}=0.85\right)$ and $\alpha_{n}=0.5$ (right panel). The dotted lines indicate the speed of sound and the Jouguet detonation velocity.

treme in the sense that a little increase causes the phase transition to remain stuck in the high-temperature phase and the universe to enter a period of inflation. In spite of this, the consequences of such models may be interesting and deserve further consideration. The absence of stationary solutions in some ranges of parameters may be related to the existence of runaway solutions [15], which correspond to the wall propagating ultrarelativistically, with the gamma factor $\gamma$ growing linearly with time. Next we discuss this possibility.

\section{$6 \quad$ Friction saturation and runaway solutions}

The equation for the friction, Eq. (2), is obtained from the equation of motion for the field $\phi$,

$$
\partial_{\mu} \partial^{\mu} \phi+\frac{\partial V}{\partial \phi}+\sum_{i} \frac{d m_{i}^{2}}{d \phi} \int \frac{d^{3} p}{(2 \pi)^{3} 2 E_{i}} f_{i}(p)=0,
$$

where $V$ is the zero-temperature effective potential and $f_{i}$ is the distribution function of particle species $i$. The latter can be decomposed into the equilibrium distribution function $f_{i}^{\text {eq }}$ and a deviation $\delta f_{i}$. The equilibrium part of $f_{i}$ in Eq. (35) gives $\partial V_{T} / \partial \phi$, where $V_{T}$ is the thermal part of the finite-temperature effective potential (i.e., $\mathcal{F}=V+V_{T}$ ). Together with the term $\partial V / \partial \phi$ this gives $\partial \mathcal{F} / \partial \phi$, which is the second term in Eq. (2), whereas the deviation gives the friction term. Usually, the deviations from equilibrium are assumed to be small, corresponding to a non-relativistically moving wall. This gives a friction force 
proportional to the velocity of the wall with respect to the plasma. Such microphysical calculations can be used to determine the value of the coefficient $\tilde{\eta}$ and the function $f(\phi / T)$ in Eqs. (2) and (5). In this section we shall set for simplicity $f(\phi / T)=1$. Therefore, this procedure gives a friction force per unit surface area

$$
\frac{F_{\mathrm{fr}}}{A}=\tilde{\eta} T \sigma v \equiv \eta v
$$

as explained at the end of section 2, Here, $v$ is the (negative) fluid velocity in the wall frame. This corresponds to a friction term $\tilde{\eta} T v \partial_{x} \phi$ in the field equation (5). The simplest relativistic generalization is the $u^{\mu} \partial_{\mu} \phi$ term in Eq. (2) .

Recently, the opposite limit was considered in Ref. [15]. The friction acting on the electroweak bubble wall was derived for a wall which is already propagating ultrarelativistically, with gamma factor $\gamma \sim 10^{9}$. Such a fast moving wall validates a number of approximations. The reflection coefficients are exponentially suppressed. In the frame of the wall, incoming particles have received no signal that the wall is approaching and are in equilibrium. Furthermore, interactions or scatterings between plasma particles can be neglected and the occupancies evolve undisturbed. Therefore, only the equilibrium occupancies in the symmetric phase are used in the calculation. The occupancies are assumed to be constant along a classical trajectory through the bubble wall.

As a consequence, the resulting force on the wall does not have a velocity-dependent term. The net force per unit area on the wall is found to be given by

$$
\frac{F}{A}=V\left(\phi_{+}\right)-V\left(\phi_{-}\right)-\sum_{i}\left[m_{i}^{2}\left(\phi_{-}\right)-m_{i}^{2}\left(\phi_{+}\right)\right] \int \frac{d^{3} p}{(2 \pi)^{3} 2 E_{i+}} f_{i+}^{\mathrm{eq}}(p) .
$$

This means that the total force per unit area is given by the "pressure difference"

$$
\tilde{p}_{-}-\tilde{p}_{+}=-\tilde{\mathcal{F}}_{-}+\tilde{\mathcal{F}}_{+},
$$

where $\tilde{\mathcal{F}}(\phi, T)$ is the mean field approximation to the effective potential, which is obtained by keeping only the quadratic terms in a Taylor expansion of $V_{T}$ about $\phi_{+}$[15, 16]

$$
\tilde{\mathcal{F}}(\phi, T)=V(\phi)+V_{T}\left(\phi_{+}\right)+\left.\sum_{i}\left[m_{i}^{2}(\phi)-m_{i}^{2}\left(\phi_{+}\right)\right] \frac{d V_{T}}{d m_{i}^{2}}\right|_{\phi_{+}} .
$$

To determine whether or not the wall can run away, $\tilde{\mathcal{F}}$ must be used instead of $\mathcal{F}$, and the total force (38) must be positive, i.e., if replacing $V_{T}(\phi)$ with its second-order Taylor approximation removes the minimum $\phi_{-}$or raises it above the minimum $\phi_{+}$, the bubble wall cannot run away [15].

In particular, in Ref. [15] it is shown that the bubble wall never runs away in a "fluctuation induced" first-order phase transition, i.e., a phase transition which is firstorder due to the thermal part of the potential (e.g. the MSSM). As a simple example, consider the high-temperature expansion

$$
V_{T}(\phi)=\sum_{i} \frac{T^{2} m_{i}^{2}(\phi)}{24}-\frac{T m_{i}^{3}(\phi)}{12 \pi}+\mathcal{O}\left(m^{4}\right) .
$$


It is well known that the cubic term in (40) may cause a first-order phase transition. This term is not present in the mean field potential. If the first-order character of the phase transition is due only to this term, then in the mean field potential the "broken symmetry" minimum $\phi_{-} \neq 0$ raises above the "symmetric" minimum $\phi_{+}=0$. In such a model the wall will reach a terminal velocity $v_{w}<1$. An example of a model which does not rely on the terms $T m_{i}^{3}(\phi)$ to yield a first-order phase transition is a potential with tree-level cubic terms. This is possible, e.g. in extensions of the Standard Model with singlet scalar fields, as considered in Ref. [15].

In this regime the friction force does not depend on the velocity of the fluid relative to the wall, whereas for $v \rightarrow 0$ it is proportional to $v$. It would be important for applications to know the behavior of the friction for intermediate velocities. Unfortunately, this constitutes a nontrivial problem. A simple interpolation between the two regimes was considered in Ref. [16]. The approximation consisted in the replacement $u^{\mu} \partial_{\mu} \phi \rightarrow u^{\mu} \partial_{\mu} \phi / \sqrt{1+\left(\lambda_{\mu} u^{\mu}\right)^{2}}$ in Eq. (2), with $\lambda_{\mu}=(0,0,0,1)$ in the wall frame. This is equivalent to the replacement $v \gamma \partial_{x} \phi \rightarrow v \partial_{x} \phi$. This modification does not alter the discussion of section 2. Furthermore, it simplifies the analytic equations of section 3 through the replacement $\left|v_{+}\right| \gamma_{+}+\left|v_{-}\right| \gamma_{-} \rightarrow\left|v_{+}\right|+\left|v_{-}\right|$in Eq. (25).

This phenomenological approach for the friction changes significantly the behavior in the detonation regime. As an example, we show in Fig. 8 how the results of Fig. 5 are modified. Notice that detonations exist only in a small interval of the friction parameter.

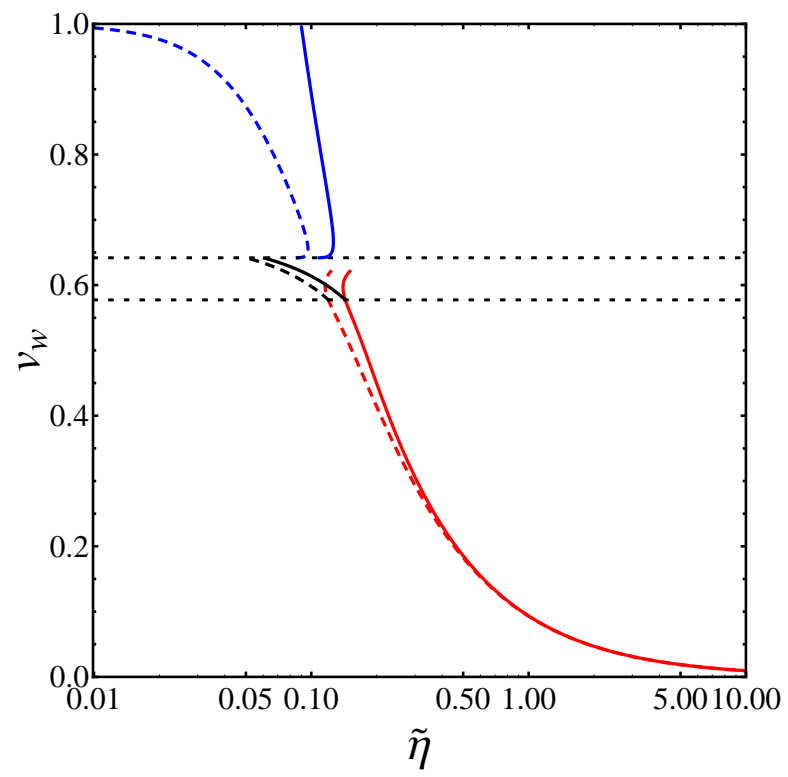

Figure 8: The wall velocity for the parameters of Fig. 5, with a friction force given by $\eta v$ (solid) and $\eta \gamma v$ (dashed). The dashed line is the curve of Fig. 5.

For higher values of the friction we have deflagrations, whereas for smaller values there is no stationary solution and the bubble wall runs away. These results are in accordance with those of Ref. [16]. In particular, we obtain detonations only in a narrow region in 
the $\eta \alpha_{n}$-plane.

On the other hand, the modification of the friction term does not affect significantly the deflagration solutions, as expected. In the previous section we have found regions of parameters corresponding to deflagrations, where there is no stationary solution (see Fig. 7). In particular, the situation of the left panel of Fig. 7 may arise in some very strongly first-order phase transitions. With the modified friction, this behavior remains (see Fig. 9). This is due to the fact that the deflagration solutions are not significantly altered by the modification of the friction. The runaway solutions appear instead for smaller values of the friction, corresponding to detonations. Nevertheless, the no-solution region has shrunk with this approximation, and it is possible that for a better approximation it will not exist at all.

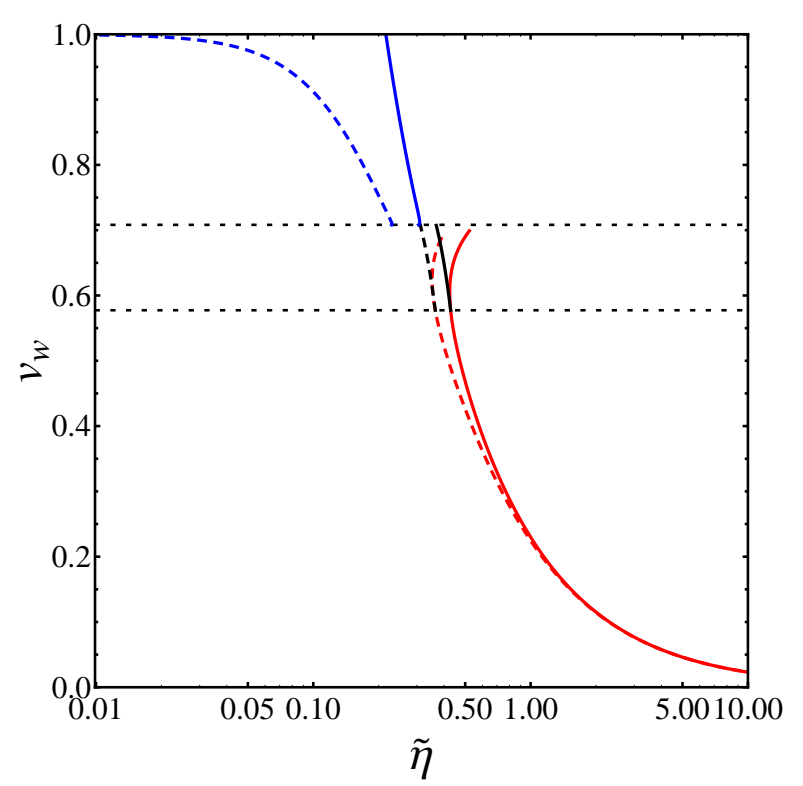

Figure 9: The wall velocity as a function of $\tilde{\eta}$ for $\alpha_{c}=4.45 \times 10^{-3}$ and $T_{n} / T_{c}=0.6$, with a friction force given by $\eta v$ (solid) and $\eta \gamma v$ (dashed).

We wish to stress that this approximation, which consists essentially in assuming a friction force of the form (36) for any wall velocity, may be too simplistic. Although it reproduces the saturation of the friction force at large $\gamma v$, the friction force saturates too soon (at values $\gamma \sim 1$ ) to its ultra-relativistic value (which is in principle valid for $\gamma \sim 10^{9}$ ). A different variation of the friction may allow the existence of stationary solutions (i.e., detonations) for a wider range of parameters. In particular, it is not clear that the friction should be a monotonically growing function of $v$. Indeed, the assumptions that lead to the runaway solution are based on the fact that the fluid is rather unaffected by the passage of the wall, due to the high speed of the latter. Conversely, the fact that the wall is unaffected by the fluid allows it to accelerate. This is somewhat similar to what happens at the macroscopic level with the stationary hydrodynamical modes, namely, the fastest the detonation, the weaker the disturbance it causes on the fluid [16, 17]. Moreover, 
solutions with intermediate velocities (around the Jouguet point) cause the maximum disturbance. Weaker deflagrations have small velocities and cause little perturbations. Weaker detonations cause less perturbations of the fluid and can thus move much faster. We may expect a similar behavior at the microscopic level, i.e., that intermediate velocities will cause larger departures from the equilibrium distributions and, thus, a higher friction.

In particular, it may happen that the real friction force is well approximated by $\eta \gamma v$ up to relatively high values of $\gamma v$, such that $\gamma \gg 1$ but not yet as large as to fulfill the hypothesis of Ref. [15]. If this is the case, then the wall will end up moving with a terminal velocity with a moderate value of $\gamma$ and never reach the ultra-relativistic regime, in spite of the existence of runaway solutions. In such a case, the model $F_{\text {fr }} / A=\eta \gamma v$ considered in the previous section would give a better approximation than $\eta v$, although the latter gives the correct ultra-relativistic behavior. Furthermore, it seems that this approximation does not take into account the fact that, if the first-order nature of the phase transition is fluctuation induced, then the bubble wall should not run away, even if the friction coefficient is very small. In such a case we expect the dashed line in Fig. 8 to give the correct behavior.

\section{Conclusions}

We have investigated the steady state motion of phase-transition fronts in a cosmological first-order phase transition. Our main goal was to find analytical approximations for the wall velocity, taking into account the different possibilities for the hydrodynamical modes and fluid profiles. Therefore, we have considered the case of planar walls, which allow to obtain analytical approximations. In Ref. [14] we considered the cases of weak deflagrations preceded by a shock front and weak detonations followed by a rarefaction wave. Here, we have studied also the case of Jouguet deflagrations which have both shock and rarefaction waves and move supersonically. We have considered two different phenomenological models for the friction. One of them grows linearly with the relativistic velocity $\gamma v$ [10, and the other saturates for large $\gamma v$ [16]. The latter reproduces the behavior of the friction force in the ultra-relativistic limit and leads to runaway solutions [15].

Our main result is a set of algebraic equations which allow to obtain, from the thermodynamic parameters and the friction coefficient, the value of the wall velocity which will be realized as the final stationary state. These analytical results rely on several approximations, such as the use of the bag equation of state and the thin wall approximation. Implementing the latter in the equation for the friction requires some ansatz for the variation of the entropy density inside the wall and also for that of the fluid velocity. The approximation for the entropy density seems to be the roughest one, as we do not obtain the curve of zero entropy production in the limit of vanishing friction. By comparing with numerical lattice calculations [10, 11, 12, we have checked that the strongest departure from the exact solution occurs for the strongest physical solutions, i.e., those around the Jouguet points (either for detonations or deflagrations). 
For a friction of the form $\gamma v$, our results are in good quantitative agreement with the cases of planar walls considered in Refs. [10, 11] and in good qualitative agreement with those of spherical bubbles considered in Refs. [11, 12]. For a friction which saturates for large $\gamma$, the wall velocity shows essentially the same behavior as in Ref. [16], which considered spherical bubbles. We remark that this latter approximation, although reproducing the correct behaviors for $v \rightarrow 0$ and for $v \rightarrow 1$, may still be too simplistic for intermediate velocities. In particular, the friction saturates to a constant value for relatively small velocities, i.e., for values of the gamma factor which are much smaller than those which justify the approximations that lead to the runaway solution [15]. As a consequence, the region of parameter space in which the bubble wall runs away may be largely overestimated. The problem of determining the behavior of the friction at intermediate velocities is a difficult one and deserves further investigation, since it has important implications for cosmology.

\section{Acknowledgements}

This work was supported in part by Universidad Nacional de Mar del Plata, Argentina, grants EXA 473/10 and 505/10. The work by A.M. was supported by CONICET through project PIP 112-200801-00943. The work by A.D.S. was supported by CONICET through project PIP 122-201009-00315.

\section{References}

[1] A. H. Guth and E. J. Weinberg, Phys. Rev. D 23, 876 (1981).

[2] G. W. Anderson and L. J. Hall, Phys. Rev. D 45, 2685 (1992).

[3] A. Megevand, Int. J. Mod. Phys. D 9, 733 (2000) hep-ph/0006177]; A. Megevand, Phys. Rev. D 64, 027303 (2001) [hep-ph/0011019]. A. Megevand, Phys. Rev. D 69, 103521 (2004); A. Megevand and A. D. Sanchez, Phys. Rev. D 77, 063519 (2008) arXiv:0712.1031 [hep-ph]].

[4] For reviews, see A. G. Cohen, D. B. Kaplan and A. E. Nelson, Ann. Rev. Nucl. Part. Sci. 43, 27 (1993) arXiv:hep-ph/9302210; A. Riotto and M. Trodden, Ann. Rev. Nucl. Part. Sci. 49, 35 (1999) [arXiv:hep-ph/9901362].

[5] E. Witten, Phys. Rev. D 30, 272 (1984); G. M. Fuller, G. J. Mathews and C. R. Alcock, Phys. Rev. D 37, 1380 (1988); J. H. Applegate and C. J. Hogan, Phys. Rev. D 31, 3037 (1985); H. Kurki-Suonio, Phys. Rev. D 37, 2104 (1988); J. Ignatius, K. Kajantie, H. Kurki-Suonio and M. Laine, Phys. Rev. D 50, 3738 (1994) arXiv:hep-ph/9405336]; A. F. Heckler, Phys. Rev. D 51 405, (1995) |arXiv:astro-ph/9407064|; A. Mégevand and F. Astorga, Phys. Rev. D 71, 023502 (2005). 
[6] D. Grasso and H. R. Rubinstein, Phys. Rept. 348, 163 (2001) arXiv:astro-ph/0009061].

[7] A. Vilenkin and E.P.S. Shellard, Cosmic Strings and Other Topological Defects (Cambridge University Press, Cambridge, England, 1994); A. Vilenkin, Phys. Rept. 121, 263 (1985).

[8] A. Kosowsky and M. S. Turner, Phys. Rev. D 47, 4372 (1993); M. Kamionkowski, A. Kosowsky and M. S. Turner, Phys. Rev. D 49, 2837 (1994); A. Kosowsky, A. Mack and T. Kahniashvili, Phys. Rev. D 66, 024030 (2002); A. D. Dolgov, D. Grasso and A. Nicolis, Phys. Rev. D 66, 103505 (2002); C. Caprini and R. Durrer, Phys. Rev. D 74, 063521 (2006); C. Caprini, R. Durrer and G. Servant, Phys. Rev. D 77, 124015 (2008) arXiv:0711.2593 [astro-ph]]; R. Apreda, M. Maggiore, A. Nicolis and A. Riotto, Nucl. Phys. B 631, 342 (2002); A. Nicolis, Class. Quant. Grav. 21, L27 (2004); C. Grojean and G. Servant, Phys. Rev. D 75, 043507 (2007); S. J. Huber and T. Konstandin, JCAP 0809, 022 (2008) arXiv:0806.1828 [hep-ph]]; S. J. Huber and T. Konstandin, JCAP 0805, 017 (2008) arXiv:0709.2091 [hep-ph]]. A. Megevand, Phys. Rev. D 78 (2008) 084003 arXiv:0804.0391 [astro-ph]]; J. Kehayias and S. Profumo, JCAP 1003, 003 (2010) [arXiv:0911.0687 [hep-ph]].

[9] L. Leitao, A. Megevand and A. D. Sanchez, arXiv:1205.3070 [astro-ph.CO].

[10] J. Ignatius, K. Kajantie, H. Kurki-Suonio and M. Laine, Phys. Rev. D 49, 3854 (1994).

[11] H. Kurki-Suonio and M. Laine, Phys. Rev. D 51, 5431 (1995) arXiv:hep-ph/9501216.

[12] H. Kurki-Suonio and M. Laine, Phys. Rev. D 54, 7163 (1996) hep-ph/9512202].

[13] S. R. Coleman, Phys. Rev. D 15, 2929 (1977) [Erratum-ibid. D 16, 1248 (1977)]; C. G. Callan and S. R. Coleman, Phys. Rev. D 16, 1762 (1977); I. Affleck, Phys. Rev. Lett. 46, 388 (1981); A. D. Linde, Nucl. Phys. B 216, 421 (1983) [Erratum-ibid. B 223, 544 (1983)]; Phys. Lett. B 100, 37 (1981).

[14] A. Megevand and A. D. Sanchez, Nucl. Phys. B 820, 47 (2009) arXiv:0904.1753 [hep-ph]].

[15] D. Bodeker and G. D. Moore, JCAP 0905, 009 (2009) [arXiv:0903.4099 [hep-ph]].

[16] J. R. Espinosa, T. Konstandin, J. M. No and G. Servant, JCAP 1006, 028 (2010) arXiv:1004.4187 [hep-ph]];

[17] L. Leitao and A. Megevand, Nucl. Phys. B 844, 450 (2011) arXiv:1010.2134 [astroph.CO]].

[18] T. Konstandin and J. M. No, JCAP 1102, 008 (2011) arXiv:1011.3735 [hep-ph]]. 
[19] M. Gyulassy, K. Kajantie, H. Kurki-Suonio and L. D. McLerran, Nucl. Phys. B 237, 477 (1984); K. Enqvist, J. Ignatius, K. Kajantie and K. Rummukainen, Phys. Rev. D 45, 3415 (1992); H. Kurki-Suonio, Nucl. Phys. B 255, 231 (1985); K. Kajantie and H. Kurki-Suonio, Phys. Rev. D 34, 1719 (1986);

[20] A. Megevand and A. D. Sanchez, Nucl. Phys. B 825, 151 (2010) arXiv:0908.3663 [hep-ph]].

[21] B. H. Liu, L. D. McLerran and N. Turok, Phys. Rev. D 46, 2668 (1992); N. Turok, Phys. Rev. Lett. 68, 1803 (1992); M. Dine, R. G. Leigh, P. Y. Huet, A. D. Linde and D. A. Linde, Phys. Rev. D 46, 550 (1992) arXiv:hep-ph/9203203]; S. Y. Khlebnikov, Phys. Rev. D 46, 3223 (1992); P. Arnold, Phys. Rev. D 48, 1539 (1993) arXiv:hep-ph/9302258; G. D. Moore and T. Prokopec, Phys. Rev. D 52, 7182 (1995) arXiv:hep-ph/9506475]; Phys. Rev. Lett. 75, 777 (1995) [arXiv:hep-ph/9503296]; P. John and M. G. Schmidt, Nucl. Phys. B 598, 291 (2001) [Erratum-ibid. B 648, 449 (2003)]; G. D. Moore, JHEP 0003, 006 (2000); G. D. Moore and N. Turok, Phys. Rev. D 55, 6538 (1997) arXiv:hep-ph/9608350.

[22] L. D. Landau and E. M. Lifshitz, Fluid Mechanics (Pergamon Press, New York, 1989); R. Courant and K. O. Friedrichs, Supersonic Flow and Shock Waves (Springer-Verlag, Berlin, 1985). 